\title{
Cost effectiveness of telehealth for patients with long term conditions (Whole Systems Demonstrator telehealth questionnaire study): nested economic evaluation in a pragmatic, cluster randomised controlled trial
}

The authors of this paper, Catherine Henderson and colleagues, have told us of some transcription errors they made in the tables (BMJ 2013;346:f1035, doi:10.1136/bmj.f1035). In table 1 $\Downarrow$ the raw differences for use of "GP (home)", "GP (surgery)", and

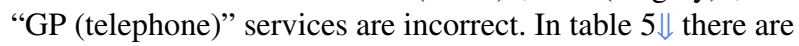
errors in the QALY value for the primary outcome in the telehealth group; the ICER for CESD-10; and the footnote text. There are also errors scattered throughout table $4 \Downarrow$. The three corrected tables are here.

Cite this as: BMJ 2013;346:f2065

๑ BMJ Publishing Group Ltd 2013 


\section{Tables}

Table 1] [CORRECTED VERSION] Self reported service use (contacts) and unit costs to be applied, across treatment groups at 12 month follow-up

\begin{tabular}{|c|c|c|c|c|c|c|}
\hline \multirow[b]{2}{*}{ Service use item } & \multicolumn{2}{|c|}{ Mean (SE) contacts ${ }^{*}$} & \multicolumn{2}{|c|}{ Between group difference } & \multirow[b]{2}{*}{ Unit cost $(£)$} & \multirow[b]{2}{*}{ Unit } \\
\hline & Usual care $(n=431)$ & Telehealth $(n=538)$ & Raw (£) & $\begin{array}{c}\text { Standardised } \\
(\%) \dagger\end{array}$ & & \\
\hline \multicolumn{7}{|l|}{ Hospital use } \\
\hline Emergency department & $0.38(0.07)$ & $0.23(0.04)$ & $-0.15 \ddagger$ & -13.7 & 103.00-133.00 & Per attendance ${ }^{26}$ \\
\hline Inpatient bed days & $1.23(0.24)$ & $0.98(0.22)$ & -0.25 & -5.0 & $116.00-1657.00$ & Per day ${ }^{26}$ \\
\hline $\begin{array}{l}\text { Day hospital and other day } \\
\text { attendances }\end{array}$ & $0.51(0.12)$ & $0.39(0.1)$ & -0.13 & -5.4 & $156.00-1496.00$ & Per attendance ${ }^{26}$ \\
\hline Outpatient attendances & $1.26(0.13)$ & $1.07(0.08)$ & -0.18 & -8.3 & $23.00-306.00$ & Per attendance ${ }^{26}$ \\
\hline \multicolumn{7}{|c|}{ Community health services/primary care } \\
\hline Paramedic & $0.18(0.04)$ & $0.13(0.02)$ & -0.05 & -8.0 & 192.00 & Per visit ${ }^{24}$ \\
\hline Community matron (visit) & $0.76(0.15)$ & $0.7(0.14)$ & -0.06 & -1.9 & $1.31,38.00$ & Per minute, per visit ${ }^{24}$ \\
\hline Community matron (telephone) & $0.2(0.04)$ & $0.38(0.1)$ & 0.18 & 10.4 & 1.28 & Per minute ${ }^{24}$ \\
\hline $\begin{array}{l}\text { Community or district nurse } \\
\text { (visit) }\end{array}$ & $0.73(0.26)$ & $1.26(0.74)$ & 0.53 & 4.2 & $1.13,24.00$ & Per minute ${ }^{24}$ per visit ${ }^{24}$ \\
\hline $\begin{array}{l}\text { Community or district nurse } \\
\text { (telephone) }\end{array}$ & $0.14(0.06)$ & $0.24(0.07)$ & 0.10 & 6.8 & 0.52 & Per minute ${ }^{24}$ \\
\hline Practice nurse & $1.5(0.15)$ & $1.26(0.11)$ & -0.24 & -9.3 & 0.52 & Per minute $^{24}$ \\
\hline Night nurse & 0 & $0.01(0.01)$ & 0.01 & 6.1 & 0.50 & Per minute ${ }^{24}$ \\
\hline Specialist nurse & $0.69(0.1)$ & $0.64(0.08)$ & -0.05 & -2.6 & $0.95-1.31$ & Per minute ${ }^{24}$ \\
\hline $\begin{array}{l}\text { Physiotherapist or occupational } \\
\text { therapist }\end{array}$ & $0.7(0.3)$ & $0.29(0.08)$ & -0.41 & -10.9 & 0.65 & Per minute $^{24}$ \\
\hline GP (home) & $0.37(0.07)$ & $0.23(0.07)$ & -0.13 & -11.1 & $4.00,94.00$ & Per minute ${ }^{24}$ per visit \\
\hline GP (surgery) & $1.69(0.09)$ & $1.7(0.1)$ & 0.01 & 0.7 & $2.40,28.00$ & Per minute, per visit ${ }^{24}$ \\
\hline GP (telephone) & $0.52(0.07)$ & $0.42(0.04)$ & -0.10 & -10.6 & 17.00 & Per consultation ${ }^{24}$ \\
\hline Dentist & $0.42(0.06)$ & $0.45(0.05)$ & 0.03 & 3.2 & 86.85 & Contact $^{26}$ \\
\hline Chiropodist & $0.61(0.13)$ & $0.6(0.11)$ & -0.01 & -0.4 & 35.37 & Contact $^{26}$ \\
\hline Optician & $0.48(0.09)$ & $0.37(0.04)$ & -0.11 & -8.4 & 20.26 & Per eye test ${ }^{27}$ \\
\hline \multicolumn{7}{|l|}{ Community mental health } \\
\hline Psychiatrist & $0.02(0.01)$ & $0.02(0.01)$ & -0.00 & -0.2 & 4.72 & Per minute ${ }^{24}$ \\
\hline Mental health nurse & $0.02(0.01)$ & $0.03(0.02)$ & 0.01 & 3.6 & 0.83 & Per minute ${ }^{24}$ \\
\hline \multicolumn{7}{|l|}{ Community care services } \\
\hline Social worker & $0.35(0.23)$ & $0.16(0.05)$ & -0.19 & -6.0 & 0.92 & Per minute ${ }^{24}$ \\
\hline $\begin{array}{l}\text { Day and evening care/help at } \\
\text { home }\end{array}$ & $6.36(1.4)$ & $4.98(1.5)$ & -1.38 & -4.3 & 0.42 & Per minute ${ }^{24}$ \\
\hline Paid night carer & $0.19(0.11)$ & $0.4(0.24)$ & 0.21 & 4.9 & 0.50 & Per minute ${ }^{24}$ \\
\hline Meals on wheels & $0.45(0.26)$ & $0.65(0.46)$ & 0.20 & 2.3 & 5.00 & Per meal $\left.\right|^{24}$ \\
\hline Major and minor adaptations & $0.07(0.02)$ & $0.08(0.02)$ & 0.01 & 3.5 & $1.50-455$ & Per adaptation ${ }^{24}$ \\
\hline $\begin{array}{l}\text { Equipment (such as mobility, } \\
\text { ADL) }\end{array}$ & $0.17(0.04)$ & $0.19(0.03)$ & 0.02 & 2.2 & $0.20-97.5$ & Per item $24,28,29$ \\
\hline \multicolumn{7}{|l|}{ Care home respite } \\
\hline Days & $0.02(0.02)$ & $0.03(0.03)$ & 0.00 & 0.5 & $63.72-70.57$ & Per day ${ }^{24}$ \\
\hline \multicolumn{7}{|l|}{ Day services } \\
\hline $\begin{array}{l}\text { Day care and other day } \\
\text { attendances }\end{array}$ & $0.59(0.18)$ & $0.44(0.16)$ & -0.15 & -4.1 & $36.00-155.82$ & Per attendance $e^{24,26,30,31}$ \\
\hline \multicolumn{7}{|l|}{ Drug treatment } \\
\hline No of drugs & $8.57(0.23)$ & $8.64(0.2)$ & 0.07 & 1.7 & Various & Various $^{32}$ \\
\hline
\end{tabular}

$£ 1=€ 1.14 ; \$ 1.49$. Unit costs were applied to the last three months before 12 month follow-up. 
Table 1 (continued)

\begin{tabular}{|c|c|c|c|c|c|}
\hline \multirow[b]{2}{*}{ Service use item } & Mean (SE) contacts* & \multicolumn{2}{|c|}{ Between group difference } & & \multirow[b]{2}{*}{ Unit } \\
\hline & Usual care $(n=431)$ Telehealth $(n=538)$ & Raw $(£)$ & $\begin{array}{c}\text { Standardised } \\
(\%) \dagger\end{array}$ & Unit cost $(£)$ & \\
\hline
\end{tabular}

$\mathrm{SE}=$ standard error; $\mathrm{ADL}=$ activities of daily living.

*Mean contacts for all participants who completed the questionnaire within each treatment group.

$\dagger$ Standardised difference=difference between group means divided by standard deviation of the total sample.

$\ddagger P<0.05, t$ test. 
Table 5| [CORRECTED VERSION] Differences in costs ${ }^{\star}$ and effect between treatment groups at 12 month follow-up, from net benefit analyses. Data are mean ( $95 \%$ confidence interval) unless otherwise stated

\begin{tabular}{|c|c|c|c|}
\hline & Usual care $(n=431)$ & Telehealth $(n=534)$ & $\begin{array}{l}\text { Between group difference } \\
\text { or ICER }(95 \% \mathrm{Cl})\end{array}$ \\
\hline \multicolumn{4}{|l|}{ Primary outcome } \\
\hline QALY (raw mean difference) $\dagger$ & 0.549 (0.52 to 0.577$)$ & $0.560(0.535$ to 0.585$)$ & $0.012(-0.026$ to 0.049$)$ \\
\hline Cost ( $(;$ raw mean difference) $†$ & 5559 (4752 to 6366$)$ & 6384 (5688 to 7081$)$ & 826 (-689 to 2340$)$ \\
\hline QALY (adjusted mean difference)§ł & 一 & 一 & 0.012 \\
\hline Cost (£; adjusted mean difference)§ & 5401 (4498 to 6305$)$ & 6511 (5905 to 7116$)$ & $1110(-1$ to 2220$)$ \\
\hline ICER (£ per QALY)§』 & 一 & 一 & 92000 (0 to undefined) \\
\hline \multicolumn{4}{|c|}{ Costs excluding project management costs $(£)$} \\
\hline Raw mean difference $†$ & 5555 (4748 to 6362 ) & 6193 (5491 to 6895$)$ & $637(-427$ to 1702$)$ \\
\hline Adjusted mean difference§ & 5395 (4492 to 6297) & 6322 (5712 to 6933$)$ & $928(-184$ to 2040$)$ \\
\hline ICER (£ per QALY)§』 & - & - & 79000 (undefined) \\
\hline \multicolumn{4}{|l|}{ Sensitivity analyses } \\
\hline \multicolumn{4}{|l|}{ Equipment prices reduced by $50 \%$} \\
\hline Cost ( $£$; adjusted mean difference) $\S$ & 5395 (4492 to 6298) & 6174 (5566 to 6782$)$ & $779(-333$ to 1890$)$ \\
\hline ICER (£ per QALY)§ף & 一 & - & 68000 (undefined) \\
\hline \multicolumn{4}{|l|}{ Equipment prices reduced by $80 \%$} \\
\hline Cost (£; adjusted mean difference)§ & 5391 (4488 to 6295) & 5972 (5362 to 6582$)$ & $580(-532$ to 1693$)$ \\
\hline ICER (£ per QALY)§ף & - & - & 52000 (undefined) \\
\hline \multicolumn{4}{|l|}{ Operating at increased capacity } \\
\hline Cost (£; adjusted mean difference)§ & 5395 (4491 to 6299) & 6034 (5430 to 6638$)$ & $639(-471$ to 1749$)$ \\
\hline ICER (£ per QALY)§ף & 一 & 一 & 57000 (undefined) \\
\hline \multicolumn{4}{|c|}{ Operating at increased capacity and equipment prices reduced by $50 \%$} \\
\hline Cost ( $£$; adjusted mean difference)§ & 5389 (4486 to 6293$)$ & 5697 (5090 to 6304) & $308(-803$ to 1419$)$ \\
\hline ICER (£ per QALY)§ף & - & - & 31000 (undefined) \\
\hline \multicolumn{4}{|c|}{ Operating at increased capacity and equipment prices reduced by $80 \%$} \\
\hline Cost ( $£$; adjusted mean difference) $\S$ & 5386 (4482 to 6289$)$ & 5495 (4886 to 6104$)$ & $109(-1002$ to 1221$)$ \\
\hline ICER (£ per QALY)§ף & 一 & - & 12000 (undefined) \\
\hline \multicolumn{4}{|l|}{ Secondary outcomes } \\
\hline \multicolumn{4}{|l|}{ ICECAP-O } \\
\hline Raw mean difference $†$ & $0.751(0.734$ to 0.768$)$ & $0.766(0.75$ to 0.781$)$ & $0.014(-0.011$ to 0.031$)$ \\
\hline Adjusted mean difference $\ddagger$ & - & 一 & 0.012 \\
\hline $\operatorname{ICER}(£) \S \rrbracket$ & 一 & 一 & 98000 (8000 to undefined) \\
\hline \multicolumn{4}{|l|}{ Brief STAI } \\
\hline Raw mean difference $†$ & 11.495 (11.093 to 11.896$)$ & $10.694(10.347$ to 11.04$)$ & $-0.801(-1.327$ to -0.275$)$ \\
\hline Adjusted mean difference $\$ \ddagger^{\star *}$ & - & - & -0.762 \\
\hline 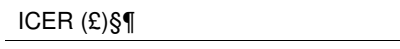 & 一 & 一 & 27000 (1000 to 86000$)$ \\
\hline \multicolumn{4}{|l|}{ CESD-10 } \\
\hline Raw mean difference $\dagger$ & 10.506 (9.882 to 11.13$)$ & 9.725 (9.17 to 10.281$)$ & $-0.781(-1.613$ to 0.052$)$ \\
\hline Adjusted mean difference§ł†† & - & 一 & -0.639 \\
\hline ICER (£)§凹 & - & - & 9000 (0 to 160000$)$ \\
\hline
\end{tabular}

\section{$£ 1=€ 1.14 ; \$ 1.49$}

${ }^{*}$ Annual equivalent costs.

†Cases for which costs data at baseline were available.

‡Derived from slope of net monetary benefit line.

§From net benefit analyses, data adjusted for baseline costs, baseline outcome, site, demographic covariates (age, sex, ethnicity, IMD, number of chronic conditions, index condition).

IRounded to nearest 1000.

${ }^{\star \star}$ Retransformed to original scale to enable comparison with raw mean difference; transformed mean $=0.042$. 


\section{Table 5 (continued)}


Table 4| [CORRECTED VERSION] Baseline characteristics of participants with available baseline economic data, at baseline and 12 month follow-up

\begin{tabular}{|c|c|c|c|c|c|c|c|c|c|c|c|c|}
\hline & \multicolumn{4}{|c|}{ Total baseline sample } & \multicolumn{4}{|c|}{$\begin{array}{l}\text { Participants completing } 12 \text { month follow-up } \\
\text { study instruments }\end{array}$} & \multicolumn{4}{|c|}{$\begin{array}{c}\text { Participants not completing } 12 \text { month } \\
\text { follow-up }\end{array}$} \\
\hline & \multirow[b]{2}{*}{$\begin{array}{c}\text { UC } \\
(n=728)\end{array}$} & \multirow[b]{2}{*}{$\begin{array}{c}\text { TH } \\
(n=841)\end{array}$} & \multicolumn{2}{|c|}{ Difference } & \multirow[b]{2}{*}{$\begin{array}{c}\text { UC } \\
(n=431)\end{array}$} & \multirow[b]{2}{*}{$\begin{array}{c}\text { TH } \\
(n=534)\end{array}$} & \multicolumn{2}{|c|}{ Difference } & \multirow[b]{2}{*}{$\begin{array}{c}\text { UC } \\
(n=297)\end{array}$} & \multirow[b]{2}{*}{$\begin{array}{c}\text { TH } \\
(n=302)\end{array}$} & \multicolumn{2}{|c|}{ Difference } \\
\hline & & & Raw $(£)$ & $\begin{array}{c}\text { Stand } \\
(\%)^{\star}\end{array}$ & & & Raw $(£)$ & $\begin{array}{c}\text { Stand } \\
(\%)^{\star}\end{array}$ & & & Raw $(£)$ & $\begin{array}{l}\text { Stand } \\
(\%)^{\star}\end{array}$ \\
\hline Age (years) & $70.6(11.8)$ & $70.1(11.8)$ & -0.46 & -4.0 & $\begin{array}{c}70.1 \\
(11.66)\end{array}$ & $\begin{array}{c}70.0 \\
(10.71)\end{array}$ & -0.12 & -1.1 & $\begin{array}{c}71.3 \\
(11.93)\end{array}$ & $\begin{array}{c}70.5 \\
(13.48)\end{array}$ & -0.78 & -6.1 \\
\hline Women (\%) & $40(n=290)$ & $41(n=347)$ & 1.4 & 2.9 & $\begin{array}{c}37.6 \\
(n=162)\end{array}$ & $\begin{array}{c}41.6 \\
(n=222)\end{array}$ & 3.9 & 8.1 & $43(n=128)$ & $41(n=124)$ & -2.0 & -4.1 \\
\hline $\begin{array}{l}\text { IMD } \\
\text { (score)§ף }\end{array}$ & $28.6(13.8)$ & $27.7(15)$ & -0.87 & -6.0 & $\begin{array}{c}27.7 \\
(13.65) \\
\end{array}$ & $26.1(14.3)$ & -1.60 & -12.0 & $\begin{array}{c}29.8 \\
(13.91) \\
\end{array}$ & $\begin{array}{c}30.6 \\
(15.75) \\
\end{array}$ & 0.72 & 4.9 \\
\hline $\begin{array}{l}\text { Group } 1 \\
(\%) \S\end{array}$ & $\begin{array}{c}17.9 \\
(n=130)\end{array}$ & $\begin{array}{c}25.6 \\
(n=215)\end{array}$ & $7.7 \ddagger$ & 18.4 & $18.9(\mathrm{n}=81)$ & $\begin{array}{c}28.2 \\
(n=151)\end{array}$ & $9.1 \ddagger$ & 21.9 & $16.5(n=49)$ & $21.2(n=64)$ & 4.7 & 12.0 \\
\hline $\begin{array}{l}\text { Group } 2 \\
(\%) \S\end{array}$ & $\begin{array}{c}22.7 \\
(n=165)\end{array}$ & $\begin{array}{c}16.8 \\
(n=141)\end{array}$ & $-5.8 \ddagger$ & -14.7 & $\begin{array}{c}24.5 \\
(n=106)\end{array}$ & $17.6(n=94)$ & $-6.8 \ddagger$ & -16.9 & $19.9(n=59)$ & $15.2(n=46)$ & -4.6 & -12.2 \\
\hline $\begin{array}{l}\text { Group } 3 \\
(\%) \S\end{array}$ & $17(n=124)$ & $\begin{array}{c}18.4 \\
(n=155)\end{array}$ & 1.4 & 3.7 & $18.4(\mathrm{n}=79)$ & $19(n=101)$ & 0.6 & 1.5 & $15.2(n=45)$ & $17.5(n=53)$ & 2.4 & 6.5 \\
\hline $\begin{array}{l}\text { Group } 4 \\
(\%) \S\end{array}$ & $\begin{array}{c}23.1 \\
(n=168)\end{array}$ & $\begin{array}{c}19.5 \\
(n=164)\end{array}$ & -3.7 & -8.8 & $20.2(n=87)$ & $\begin{array}{c}20.4 \\
(n=109)\end{array}$ & 0.1 & 0.5 & $27.3(n=81)$ & $17.9(n=54)$ & $-9.4 \ddagger$ & -22.5 \\
\hline $\begin{array}{l}\text { Group } 5 \\
(\%) \S^{\star *}\end{array}$ & $\begin{array}{c}19.4 \\
(n=141)\end{array}$ & $\begin{array}{c}19.7 \\
(n=166)\end{array}$ & 0.4 & 0.9 & $18.1(n=78)$ & $14.8(n=79)$ & -3.3 & -8.9 & $21.2(n=63)$ & $28.1(n=85)$ & $6.9 \dagger$ & 16.1 \\
\hline \multicolumn{13}{|c|}{ Index condition (\%) } \\
\hline COPD & $\begin{array}{c}33.5 \\
(n=244)\end{array}$ & $\begin{array}{c}39.7 \\
(n=334)\end{array}$ & $6.1 \dagger$ & 12.8 & $\begin{array}{c}32.5 \\
(n=140)\end{array}$ & $\begin{array}{c}43.4 \\
(n=232)\end{array}$ & $11.1 \ddagger$ & 22.5 & $35(n=104)$ & $32.8(n=99)$ & -2.2 & -4.7 \\
\hline Heart failure & $\begin{array}{c}37.8 \\
(n=275)\end{array}$ & $\begin{array}{c}31.3 \\
(n=263)\end{array}$ & $-6.5 \ddagger$ & -13.7 & $\begin{array}{c}40.6 \\
(n=175)\end{array}$ & $\begin{array}{c}33.1 \\
(n=177)\end{array}$ & $-7.8 \dagger$ & -15.5 & $\begin{array}{c}33.7 \\
(n=100)\end{array}$ & $28.5(n=86)$ & -5.2 & -11.2 \\
\hline Diabetes†† & $\begin{array}{c}28.7 \\
(n=209)\end{array}$ & $29(n=244)$ & -0.3 & 0.7 & $\begin{array}{c}26.9 \\
(n=116)\end{array}$ & $\begin{array}{c}23.4 \\
(n=125)\end{array}$ & -3.4 & -8.1 & $31.3(n=93)$ & $\begin{array}{c}38.7 \\
(n=117)\end{array}$ & 7.4 & 15.6 \\
\hline $\begin{array}{l}\text { No of } \\
\text { comorbidities }\end{array}$ & $\begin{array}{c}2 \\
(1.9) \\
\end{array}$ & $\begin{array}{c}1.8 \\
(1.8) \\
\end{array}$ & -0.18 & -9.7 & $\begin{array}{c}2 \\
(1.9) \\
\end{array}$ & $\begin{array}{c}1.8 \\
(1.8) \\
\end{array}$ & -0.22 & -12.0 & $\begin{array}{c}2.1 \\
(1.8) \\
\end{array}$ & $\begin{array}{c}2 \\
(1.8) \\
\end{array}$ & -0.09 & -4.9 \\
\hline $\begin{array}{l}\text { Baseline } \\
\text { costs }(£)\end{array}$ & $\begin{array}{c}1276 \\
(1628) \\
\end{array}$ & $\begin{array}{c}1244 \\
(1687) \\
\end{array}$ & -32 & -1.9 & $\begin{array}{c}1096 \\
(1408) \\
\end{array}$ & $\begin{array}{c}1172 \\
(1620)\end{array}$ & 92 & 5.0 & $\begin{array}{c}1536 \\
(1875) \\
\end{array}$ & $\begin{array}{c}1341 \\
(1751) \\
\end{array}$ & -195 & -10.7 \\
\hline \multicolumn{13}{|l|}{ WSD site (\%) } \\
\hline Site 1 & $\begin{array}{c}32.1 \\
(n=234)\end{array}$ & $\begin{array}{c}30.4 \\
(n=256)\end{array}$ & -1.7 & -1.9 & $\begin{array}{c}30.6 \\
(n=132)\end{array}$ & $\begin{array}{c}32.6 \\
(n=174)\end{array}$ & 1.8 & 4.2 & $\begin{array}{c}34.3 \\
(n=102)\end{array}$ & $26.8(n=81)$ & $-7.5 \dagger$ & -16.3 \\
\hline Site 2 & $\begin{array}{c}38.9 \\
(n=283)\end{array}$ & $\begin{array}{c}40.7 \\
(n=342)\end{array}$ & 1.8 & 2.0 & $\begin{array}{c}42.7 \\
(n=184)\end{array}$ & $\begin{array}{c}44.2 \\
(n=236)\end{array}$ & 1.3 & 3.0 & $33.3(n=99)$ & $\begin{array}{c}34.8 \\
(n=105)\end{array}$ & 1.4 & 3.0 \\
\hline Site 3ł‡ & $29(n=211)$ & $\begin{array}{c}28.9 \\
(n=243)\end{array}$ & -0.2 & -0.2 & $\begin{array}{c}26.7 \\
(n=115)\end{array}$ & $\begin{array}{c}23.2 \\
(n=124)\end{array}$ & -3.1 & -8.0 & $32.3(n=96)$ & $\begin{array}{c}38.4 \\
(n=116)\end{array}$ & 6.1 & 12.7 \\
\hline $\begin{array}{l}\text { White British } \\
\text { ethnicity }(\%) \S\end{array}$ & $\begin{array}{c}86.3 \\
(n=628)\end{array}$ & $\begin{array}{c}86.9 \\
(n=731)\end{array}$ & 0.6 & 1.7 & $\begin{array}{c}87.5 \\
(n=377)\end{array}$ & $\begin{array}{c}89.5 \\
(n=478)\end{array}$ & 2.0 & 6.4 & $\begin{array}{c}84.5 \\
(n=251)\end{array}$ & $\begin{array}{c}82.9 \\
(n=250)\end{array}$ & -1.6 & -4.3 \\
\hline
\end{tabular}

$£ 1=€ 1.14 ; \$ 1.49$. Data are mean (standard deviation) or proportion (\%) and no of patients.

$\mathrm{UC}=$ usual care; $\mathrm{TH}=$ telehealth; Stand=standardised difference; COPD=chronic obstructive pulmonary disease.

*Standardised difference=difference between group means divided by standard deviation of the total sample.

$\dagger \mathrm{P}<0.05$ on $z$ test of proportions.

$\ddagger P<0.01$ on $z$ test of proportions.

§Imputed data.

ףDifference between means of TH group sample at baseline and 12 month follow-up: $\mathrm{P}<0.05, \mathrm{t}=2.09$ (unpaired $t$ test).

** Difference between proportions of patients in TH group at baseline and 12 month follow-up: $Z=2.34, P<0.05$.

††Difference between proportions of patients in TH group at baseline and 12 month follow-up: $z=2.29, P<0.05$.

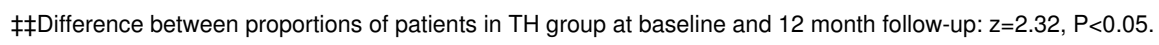

